\title{
Recht und Ethik im digitalen Zeitalter
}

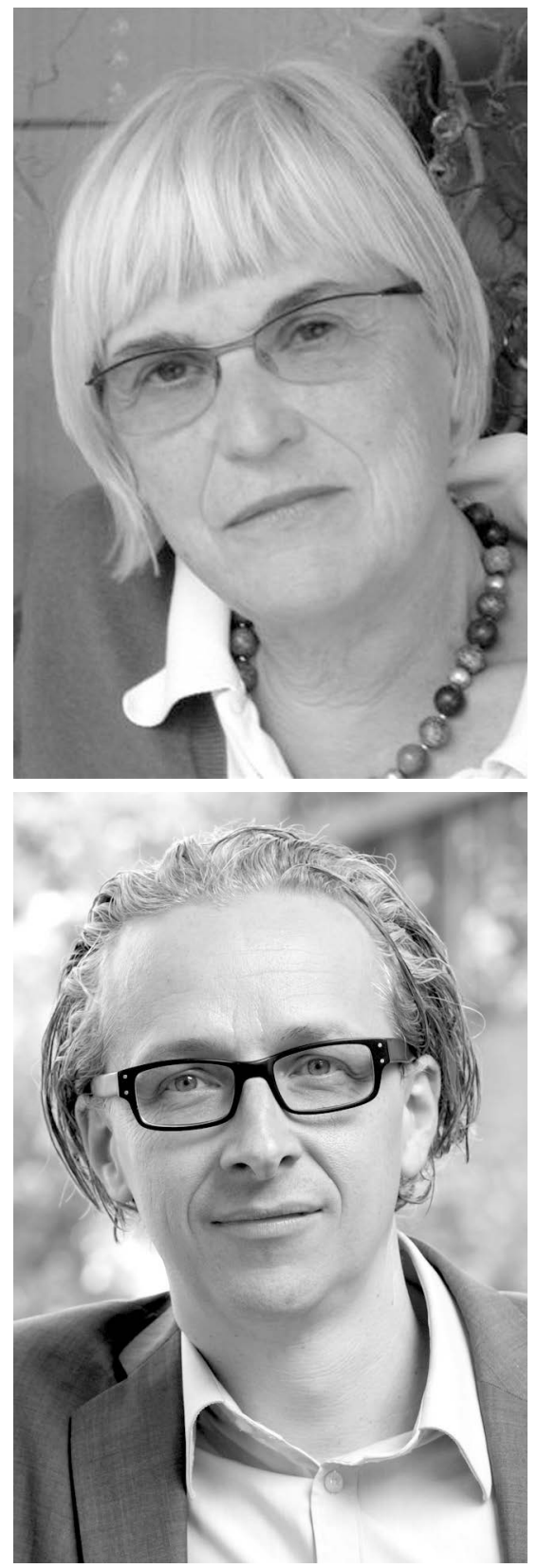

Die Geschichte der Menschheit kann auf viele Arten dargestellt werden. Der Europahistoriker Wolfgang Schmale zeichnet sie im Lichte der Freiheitsrechte nach und geht dabei insbesondere auf die Geschichte des Rechts auf Privatheit ein, das in der Epoche, die als „Enlightenment" oder Aufklärung bezeichnet wird, zunehmend Konturen als Menschenrecht und als Bastion gegen Diskriminierung und Gewalt gewinnt. An der epochalen Schwelle zum virtuellen Raum der digitalen Welt hält Schmale inne und stellt die Frage nach dem Überleben des Rechts auf Privatheit und seinen datenschutzrechtlichen Bedingungen. Diese Frage führt pfeilgerade zu Aspekten der menschlichen Verantwortung beim Einsatz Künstlicher Intelligenz (KI). Auf der 97. Datenschutzkonferenz im Hambacher Schloss haben die Datenschutzaufsichtsbehörden des Bundes und der Länder in ihrer »Hambacher Erklärung « betont, dass beim Einsatz Künstlicher Intelligenz die Menschenwürde und das in ihr verankerte Grundrecht auf informationelle Selbstbestimmung zu beachten, Diskriminierungen zu vermeiden und die klare Zurechnung von Verantwortlichkeiten erforderlich seien. Diesen Fragen widmet sich der Technikphilosoph Klaus Kornwachs am Beispiel der Intelligenten Robotik bzw. smart robots und stellt fest, dass hier Verantwortungslücken auftreten können und sich möglicherweise ein Subjekt der Verantwortung nicht mehr feststellen lässt, etwa wenn nach dem Start keine Kontrolle mehr über das System möglich ist. Kornwachs befasst sich intensiv mit den ethisch verantwortbaren Spielregeln im Vorfeld des Einsatzes von KI.

Die DS-GVO befasst sich unter dem Aspekt des Privatheitsschutzes mit besonderen Diskriminierungsrisiken (Art. 9 Abs. 1 DS-GVO). Die geschlechtsbezogene Diskriminierung und Gewalt sind Gegenstand des Beitrags von Volker Boehme-Neßler. Diese Gewalt kann vor allem Frauen besonders hart treffen (Art. 3 lit. d IK - Istanbul Konvention) und ihr Recht auf Achtung des Privat- und Familienlebens (Art. 8 EMRK und Art. 7 und 8 GRCh) verletzen. Boehme-Neßler geht dieser Frage sensibel nach und untersucht sie im Rahmen des deutschen Prostituiertenschutzgesetzes und der datenschutzrechtlichen Vorgaben der DS-GVO.

In einer weiteren Entschließung der 97. Datenschutzkonferenz ist der Bundesgesetzgeber aufgefordert worden, die europarechtlich vorgeschriebene Verhängung von Geldbußen gegen Unternehmen für Verstöße ihrer Mitarbeiter im deutschen Recht sicherzustellen. Allerdings sind Geldbußen künftig keineswegs das einzige Mittel, um dem Datenschutzrecht zu mehr Durchsetzungskraft zu verhelfen. Jürgen Kühling und Florian Sackmann lenken den Blick auf eine ganz andere Variante der Rechtsdurchsetzung, die für Verbraucher beachtliche Chancen und spiegelbildlich für Unternehmen erhebliche Risiken birgt: die prozessuale Durchsetzung von Schadensersatzansprüchen mit dem Instrument der Musterfeststellungsklage. Und auch an das Modell des "Dateneigentums" werden von verschiedenster Seite immer wieder große Hoffnungen geknüpft, auf diese Weise das Recht auf informationelle Selbstbestimmung zu stärken. Anne Riechert bietet mit ihrem Beitrag einen Rundumblick zu diesem Modell und beleuchtet es als Ausschließlichkeits- und Nutzungsrecht unter den verschiedensten Facetten. Alex Makulilo schließlich führt uns am Beispiel der Telekommunikationsgesetzgebung in Tansania mit aller Nachdrücklichkeit vor Augen, wie weit staatliche Überwachung um sich greifen kann - auch wenn an sich ein Recht auf Privatheit im Verfassungsrecht verankert ist, dieses Grundrecht aber nicht durch eine umfassende Datenschutzgesetzgebung abgesichert ist, sondern lediglich durch sektorspezifische Datenschutzgesetze ergänzt wird.

Drei Beiträge zu aktuellen Themen rund um die DS-GVO runden das Heft ab. Volker Lüdemann und Patrick Pokrant gehen der Frage nach, welche Auswirkungen die Regelungen der DS-GVO auf die Einwilligung in die Datenverarbeitung im Rahmen des digitalen Strommesswesens (sog. Smart Metering) haben. Im Forum macht uns Dennis-Kenji Kipker in seinem Beitrag zum aktuell im Gesetzgebungsprozess befindlichen Zweiten Datenschutzanpassungsgesetz nur wenig Hoffnung, dass sich mit diesem Gesetz das Datenschutzrecht hierzulande verbessern wird. Und Maximilian Schnebbe geht in der Rubrik Good Practice der Frage nach, wann eine Datenverarbeitungstechnologie so neu im Sinne der DS-GVO ist, dass eine Datenschutzfolgeabschätzung durchgeführt werden muss.

\section{Marie-Theres Tinnefeld und Benedikt Buchner}

